\title{
Genetic variants on $17 q 21$ are associated with asthma in a Han Chinese population
}

\author{
F.-X. Li ${ }^{1 *}$, J.-Y. Tan ${ }^{2 *}$, X.-X. Yang ${ }^{1}$, Y.-S. Wu ${ }^{1}, \mathrm{D} . \mathrm{Wu}^{3}$ and M. $\mathrm{Li}^{1}$ \\ ${ }^{1}$ School of Biotechnology, Southern Medical University, Guangzhou, \\ P.R. China \\ ${ }^{2}$ Central ICU of Boai Hospital of Zhongshan City Affiliated Hospital, \\ Southern Medical University, Zhongshan, P.R. China \\ ${ }^{3}$ Institute of Respiratory Diseases, \\ Affiliated Hospital of Guangdong Medical College, Zhanjiang, P.R. China \\ *These authors contributed equally to this study. \\ Corresponding author: M. Li \\ E-mail: mingli2006_2006@126.com
}

Genet. Mol. Res. 11 (1): 340-347 (2012)

Received July 15, 2011

Accepted November 24, 2011

Published February 10, 2012

DOI http://dx.doi.org/10.4238/2012.February.10.5

\begin{abstract}
A genome-wide study has shown an association between SNPs located on 17q21 and asthma. Such associations have been identified in several populations, but little is known about the Han Chinese population. We conducted a case-control study in a Han Chinese population to investigate the relationship between SNPs located on 17q21 and asthma; 241 asthmatic patients and 212 healthy controls were recruited from the outpatient clinics of the Nanfang Hospital, Guangdong Province, southern China. We genotyped six SNPs (rs8067378, rs8069176, rs2305480, rs4795400, rs12603332, and rs11650680) located on 17q21 with the Sequenom MassARRAY iPLEX platform. For two of these six loci (rs2305480 and rs8067378), there was evidence of association with asthma, and there was a weak association of asthma with rs8069176. We confirm that genetic variants on $17 q 21$ are associated with asthma in the Han Chinese population.
\end{abstract}

Key words: Asthma; 17q21; Genetic variants; Susceptibility 


\section{INTRODUCTION}

Asthma is a disease of chronic airway inflammation. It is a serious public health problem throughout the world, affecting people of all ages. Its prevalence has increased during the second half of 20th century in most countries (Eder et al., 2006). Asthma is also a complex disease caused by multiple genetic and environmental factors. More than 100 genes have been reported to be associated with asthma, but few have been investigated in multiple populations (Ober and Hoffjan, 2006). In a whole-genome association study, Moffatt et al. (2007) identified a region on chromosome 17q21 that was strongly associated with asthma. Subsequently, a series of related studies were performed that highlighted this association in different ethnic groups (Sleiman et al., 2008; Madore et al., 2008; Galanter et al., 2008; Hirota et al., 2008; Tavendale et al., 2008; Leung et al., 2009). These findings suggested that orosomucoid 1 (ORM1)-like 3 (ORMDL3), gasdermin-like (GSDML), or adjacent genes located on chromosome $17 \mathrm{q} 21$ may be associated with asthma. However, these studies have mainly concentrated on Western populations. Our study aimed to investigate the associations between asthma and the identified SNPs on 17q21 in a Chinese population. In the present study, six SNPs in $G S D M L, O R M D L 3$, and the adjacent region were selected. Figure 1 shows the distribution of these six SNPs on the $17 \mathrm{q} 21$ region.

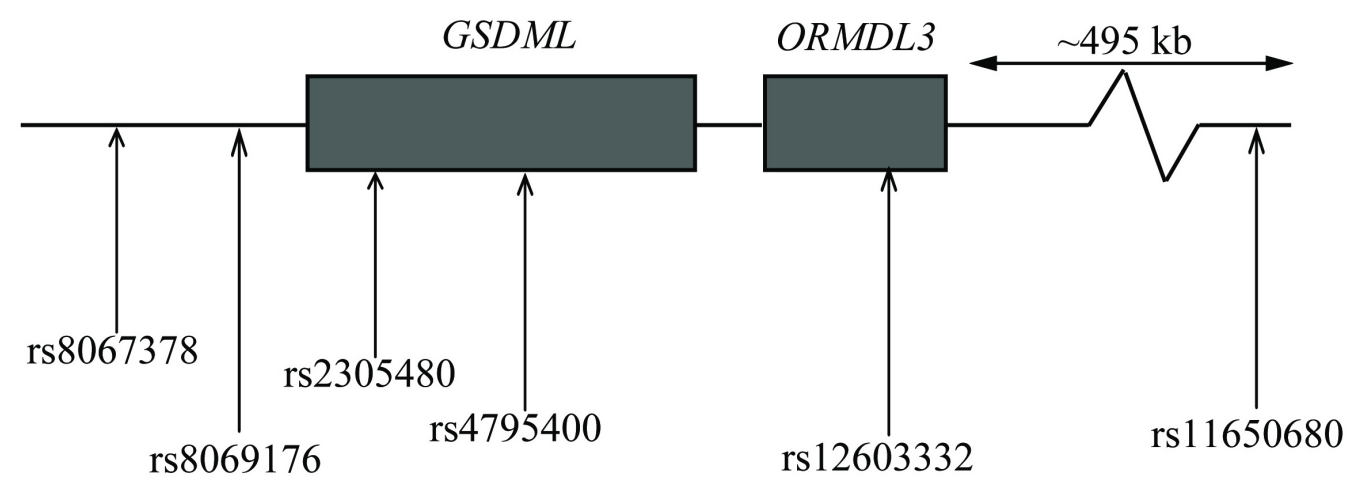

Figure 1. The distribution of six SNPs in our study along the chromosome 17q12-21 region.

\section{MATERIAL AND METHODS}

\section{Subject recruitment}

Patients and control subjects were obtained from individuals attending the outpatient clinics of Nanfang Hospital, Guangzhou, Guangdong Province, southern of China. All patients and control subjects were recruited between 2008 and 2010. The diagnosis of asthma was based on clinical symptoms, and clinical testing was performed by a physician on the basis of international asthma guidelines (GINA 2007). The control population consisted of outpatients without symptoms of asthma (wheezing, cough or dyspnea) and no diagnosis of asthma. The study protocol was approved by the Clinical Research Ethics Committee of Nanfang Hospital. Informed consent was obtained from all participants. 


\section{Genotyping}

Genomic DNA was extracted from $200 \mu \mathrm{L}$ peripheral blood using the Genomic DNA Purification kit (TIANGEN Biotech, Beijing, China) according to manufacturer instructions and stored at $-70^{\circ} \mathrm{C}$ until use. All SNPs were genotyped using the Sequenom MassARRAY matrix-assisted laser desorption/ionization time-of-flight mass spectrometry platform (Sequenom, San Diego, CA, USA). Primers were designed using a semi-automated method (Assay Design 3.1, Sequenom).

\section{Statistical analysis}

Hardy-Weinberg equilibrium (HWE), linkage disequilibrium (LD) and the pairwise LD coefficient for the six SNPs in the controls were calculated by Haploview 4.2 (Daly Lab., Cambridge, MA, USA). The differences in the genotype distributions between the cases and control subjects were evaluated using the $\chi^{2}$ test. The associations between polymorphisms and asthma risk were estimated using the odds ratio (OR) and $95 \%$ confidence intervals $(95 \% \mathrm{CI})$ using binary logistic regression analyses, controlling for age and gender as covariates. The statistical tests, haplotypes, and their associations with asthma were analyzed using the web-based tool SNPstats (http://bioinfo.iconcologia.net/SNPstats). The results obtained with this calculator were consistent with the SPSS 13.0 software and have been summarized in another publication (Li et al., 2011). All comparisons were two-sided, and $\mathrm{P}<0.05$ was considered to be significant.

\section{RESULTS}

\section{Study population}

In total, 241 unrelated patients with bronchial asthma and 212 control individuals were enrolled. The patient group consisted of 118 men and 123 women, with an average age of 41.48 years $(\mathrm{SD}=16.03$; range $=11-80$ years $)$. The control group consisted of 160 men and 52 women, with an average of 40.65 years $(S D=20.29$; range $=18-89$ years $)$. The details of the patients and control subjects are listed in Table 1.

Table 1. Characteristics of asthma patients and healthy controls.
\begin{tabular}{lcc}
\hline & Case subjects $(\mathrm{N}=241)$ & Control subjects (N=212) \\
\hline Variable & $41.98 \pm 16.17$ & $40.71 \pm 20.17$ \\
\hline Mean age (years; means \pm SD) & $48.96(118)$ & $75.47(160)^{*}$ \\
Male \% (N) & $59.88 \pm 24.30$ & ND \\
FEV1 (means \pm SD) & $76.91 \pm 84.29$ & ND \\
FVC (means \pm SD) & 62.30 & ND \\
\hline Smoking (\%)
\end{tabular}

$\mathrm{ND}=$ not done. $* \mathrm{P}<0.05$ by the $\chi^{2}$ test.

\section{Characterization of SNPs located on chromosome 17q21}

The overall genotyping efficiency was $93.0 \%$. Table 2 summarizes minor allele frequencies for the six SNPs. All SNPs followed HWE among the controls. The LD scores for 
these SNPs among our control subjects were generally high for SNPs around the GSDML and ORMDL3 genes (Figure 2). The SNP rs11650680, which is distant from the two genes, had a low LD compared to the other SNPs.

\begin{tabular}{|c|c|c|c|c|c|c|c|}
\hline \multirow[t]{2}{*}{ SNP } & \multirow[t]{2}{*}{ Minor allele } & \multicolumn{2}{|c|}{ Present study } & \multicolumn{4}{|c|}{ HapMap data } \\
\hline & & Control & Cases & Beijing & Japan & European & African \\
\hline rs8067378 & $\mathrm{G}$ & 0.19 & 0.22 & 0.38 & 0.31 & 0.51 & 0.54 \\
\hline rs8069176 & A & 0.22 & 0.20 & 0.38 & 0.31 & 0.47 & 0.36 \\
\hline rs 2305480 & $\mathrm{~T}$ & 0.03 & 0.14 & 0.38 & 0.31 & 0.47 & 0.07 \\
\hline rs4795400 & $\mathrm{T}$ & 0.25 & 0.21 & 0.38 & 0.31 & 0.48 & 0.07 \\
\hline rs12603332 & $\mathrm{T}$ & 0.29 & 0.25 & 0.41 & 0.28 & 0.48 & 0.58 \\
\hline rs11650680 & $\mathrm{T}$ & 0.23 & 0.23 & 0.20 & 0.15 & 0.24 & 0.00 \\
\hline
\end{tabular}

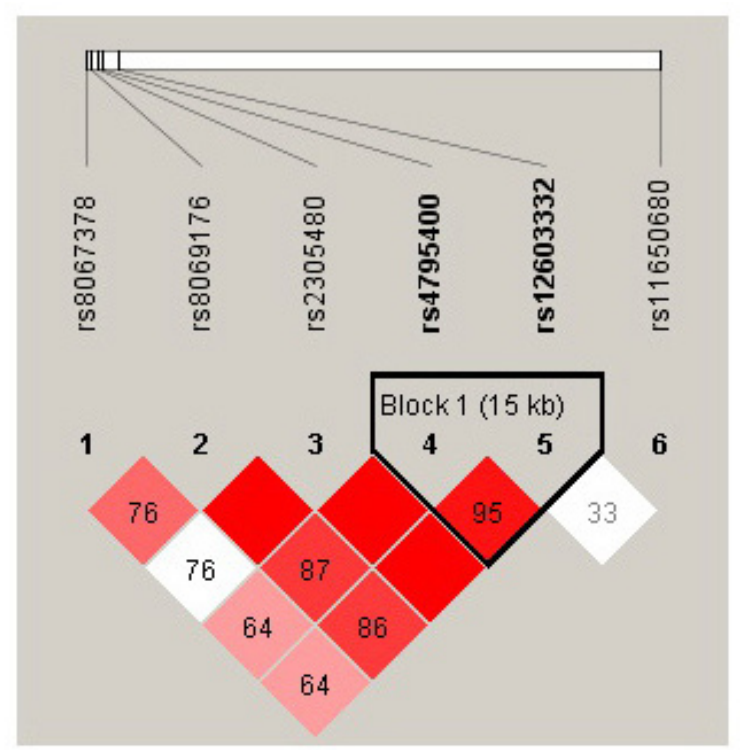

Figure 2. Linkage disequilibrium (LD) pattern among our 6 SNPs by the Haploview analysis. Numbers inside the boxes represent D' values for LD, and lacking numbers were 100 .

\section{Asthma and individual polymorphisms}

All genotype distributions for the six SNP markers in the case and control subjects are listed in Table 3. The relationship between asthma and these SNPs was analyzed by logistical regression in every chosen genetic model, and the co-dominant model is shown. Two of the six loci showed evidence of association (rs8067378 and rs2305480) with asthma. One locus, rs8069176, showed a weak trend toward an association with asthma. However, associations with asthma risk were not detected in the remaining three loci (rs4795400, rs12603332 and rs11650680). 


\begin{tabular}{|c|c|c|c|c|c|}
\hline SNP & Genotype & Control (\%) & Case (\%) & OR* $(95 \% \mathrm{CI})$ & $\mathrm{P}$ value \\
\hline \multirow[t]{3}{*}{ rs8067378 } & AA & $117(64.3)$ & $119(63.3)$ & 1.00 & \\
\hline & AG & $61(33.5)$ & $55(29.3)$ & $0.95(0.59-1.51)$ & \\
\hline & GG & $4(2.2)$ & $14(7.5)$ & $3.88(1.20-12.55)$ & 0.041 \\
\hline \multirow[t]{3}{*}{ rs8069176 } & GG & $116(58.0)$ & $145(64.4)$ & 1.00 & \\
\hline & AG & $66(33.0)$ & $71(31.6)$ & $0.94(0.61-1.44)$ & \\
\hline & AA & $18(9.0)$ & $9(4.0)$ & $0.48(0.20-1.14)$ & 0.240 \\
\hline \multirow{4}{*}{ rs 2305480} & $\mathrm{CC}$ & $193(93.7)$ & $181(78.0)$ & 1.00 & \\
\hline & CT & $13(6.3)$ & $39(16.8)$ & $3.54(1.79-7.01)$ & $<0.0001$ \\
\hline & TT & $0(0.0)$ & $12(5.2)$ & NA & \\
\hline & $\mathrm{CT}+\mathrm{TT}$ & $13(6.3)$ & $51(22.0)$ & $4.49(2.32-8.71)$ & $<0.0001$ \\
\hline \multirow[t]{3}{*}{ rs4795400 } & $\mathrm{CC}$ & $115(57.8)$ & $139(64.7)$ & 1.00 & \\
\hline & СТ & 67 (33.7) & $61(28.4)$ & $0.81(0.52-1.26)$ & \\
\hline & TT & $17(8.5)$ & $15(7.0)$ & $0.83(0.38-1.80)$ & 0.620 \\
\hline \multirow[t]{3}{*}{ rs12603332 } & $\mathrm{CC}$ & $109(52.7)$ & $138(59.2)$ & 1.00 & \\
\hline & CT & 77 (37.2) & $74(31.8)$ & $0.82(0.54-1.26)$ & \\
\hline & TT & $21(10.1)$ & $21(9.0)$ & $0.86(0.43-1.71)$ & 0.660 \\
\hline \multirow{3}{*}{ rs 11650680} & $\mathrm{CC}$ & $127(61.4)$ & $137(58.5)$ & 1.00 & \\
\hline & СT & $65(31.4)$ & $86(36.8)$ & $1.18(0.77-1.79)$ & \\
\hline & TT & $15(7.2)$ & $11(4.7)$ & $0.72(0.31-1.67)$ & 0.480 \\
\hline
\end{tabular}

$\mathrm{OR}=$ odds ratio $; 95 \% \mathrm{CI}=$ confidence interval at $95 \%$. *Adjusted for age and gender; $\mathrm{P}$ value calculated from chisquared comparison of genotype frequencies in cases versus controls. The bold values indicate $\mathrm{P}<0.05$.

The most significant difference between the case and control groups in genotype frequency was identified for the SNP rs2305480 (P < 0.0001). Compared to the CC genotype of rs2305480, the CT genotype conferred a covariate-adjusted 3.54-fold increased risk of asthma $(95 \% \mathrm{CI}=1.79-7.01)$. Because the TT genotype was not detected in the control group, the OR for TT could not be calculated. The variant homozygous CT and TT genotypes of rs 2305480 were significantly more frequent in asthmatic patients, and comparison of the CC genotype with the CT and TT genotypes revealed an increased risk of asthma $(\mathrm{OR}=4.49 ; 95 \% \mathrm{CI}=$ 2.32-8.71). The $\mathrm{T}$ allele of rs 2305480 was also associated with an increased risk of asthma $(\mathrm{OR}=4.13 ; 95 \% \mathrm{CI}=2.22-7.70)$ (data not shown). A significant difference in the genotype frequency for SNP rs 8067378 was also seen between the case and control groups $(\mathrm{P}=0.041)$. The GG carriers of rs 8067378 also showed an increased risk of asthma $(\mathrm{OR}=3.38 ; 95 \% \mathrm{CI}$ $=1.20-12.55)$ compared to the AA group. The best-fit model for $\mathrm{rs} 8067378$ was the recessive model, in which the GG genotype showed the higher risk of asthma $(\mathrm{OR}=3.96 ; 95 \% \mathrm{CI}=$ 1.24-12.65) compared to the AA and AG genotypes (data not shown).

Another SNP, rs8069176, showed a trend toward an association with asthma. The AA genotype of rs8069176 showed a decreased risk of asthma $(\mathrm{OR}=0.48 ; 95 \% \mathrm{CI}=0.20-1.14)$ compared to the GG genotype. After stratification by gender, the AA genotype of rs 8069176 showed a statistically significant decreased risk of asthma $(\mathrm{OR}=0.29 ; 95 \% \mathrm{CI}=0.09-0.90)$ among male subjects (data not shown). The remaining three SNPs were not significantly associated with asthma (rs4795400, rs12603332 and rs11650680).

\section{Asthma and haplotypes located on chromosome 17q21}

Six haplotype loci were assigned to the six SNPs in this study. Tables 4 and 5 summarize the analyses for individual haplotypes according to gender. The haplotype GACTTC and AACTTC were significantly protective against asthma, with the respective OR for the two 
phenotypes being 0.29 and 0.11 . The haplotype AACTTC was associated with a significant increase in the development of asthma, with an $\mathrm{OR}=6.95$. After stratification by gender, the statistically significant associations were only observed in male subjects for the haplotypes GACTTC and AACTTC.

Table 4. Relationship between asthma and haplotypes from six SNPs at $17 \mathrm{q} 21$ region $(\mathrm{N}=453)$.

\begin{tabular}{|c|c|c|c|c|c|}
\hline \multirow[t]{2}{*}{ Haplotype } & \multicolumn{3}{|c|}{ Frequencies } & \multirow[t]{2}{*}{$\mathrm{OR}^{*}(95 \% \mathrm{CI})$} & \multirow[t]{2}{*}{ P value } \\
\hline & Total & Control & Case & & \\
\hline AGCCCC & 0.504 & 0.497 & 0.512 & 1.00 & \\
\hline AGCCCT & 0.156 & 0.167 & 0.144 & $0.84(0.53-1.32)$ & 0.440 \\
\hline GACTTC & 0.071 & 0.116 & 0.036 & $0.29(0.12-0.72)$ & 0.008 \\
\hline AACTTC & 0.032 & 0.045 & 0.013 & $0.11(0.02-0.72)$ & 0.022 \\
\hline GATTTC & 0.031 & NA & 0.048 & $6.95(1.77-27.33)$ & 0.006 \\
\hline
\end{tabular}

$\mathrm{OR}=$ odds ratio; $95 \% \mathrm{CI}=$ confidence interval at $95 \%$. Haplotypes were constructed from the following SNPs: rs8067378, rs8069176, rs2305480, rs4795400, rs12603332, and rs11650680. Only haplotypes with $\geq 0.03$ frequencies were used. *Adjusted for age and gender. The bold values indicate $\mathrm{P}<0.05$.

\begin{tabular}{|c|c|c|c|}
\hline \multirow[t]{2}{*}{ Haplotype } & \multirow[t]{2}{*}{ Frequency } & \multicolumn{2}{|c|}{$\mathrm{OR} *(95 \% \mathrm{CI})$} \\
\hline & & Female & Male \\
\hline AGCCCC & 0.504 & 1.00 & 1.00 \\
\hline AGCCCT & 0.156 & $1.07(0.51-2.28)$ & $0.69(0.39-1.23)$ \\
\hline GACTTC & 0.073 & $0.26(0.06-1.06)$ & $0.24(0.08-0.74)$ \\
\hline AACTTC & 0.031 & $0.45(0.04-5.33)$ & $0.10(0.01-0.78)$ \\
\hline AGCTTC & 0.030 & $1.37(0.27-6.89)$ & $1.50(0.42-5.36)$ \\
\hline
\end{tabular}

$\mathrm{OR}=$ odds ratio $; 95 \% \mathrm{CI}=$ confidence interval at $95 \%$. Haplotypes were constructed from the following SNPs: rs8067378, rs8069176, rs2305480, rs4795400, rs12603332, and rs11650680. *Adjusted for age. The bold values indicate $\mathrm{P}<0.05$.

\section{DISCUSSION}

The present study provides further data to support the association between asthma and variants located on chromosome $17 \mathrm{q} 21$. In this study, the most strongly associated SNPs were rs2305480 and rs8067378, which is consistent with recent studies (Flory et al., 2009; Bisgaard et al., 2009; Halapi et al., 2010). Additionally, Bouzigon et al. (2008) reported a significant association between rs 2305480 and asthma in a French population. With regard to the SNP rs8067378, Moffatt et al. (2007) reported that rs8067378 was the most strongly associated marker for asthma in a family-based test. The association of rs 8067378 with childhood asthma was also observed in a study investigating North Americans of European ancestry (Sleiman et al., 2008).

The two associated SNPs in this study, rs 2305480 and rs8067378, are both located in GSDML, the first in an exon and the second upstream of the gene. The most prominent SNP, rs7216389, has been strongly associated with asthma in GWAS (Moffatt et al., 2007), also located in an intron of the GSDML gene. GSDML encodes a member of the gasdermin domaincontaining protein family, whose function is currently unclear. Moffatt et al. (2007) found that the polymorphisms in GSDML and the transcript levels of $O R M D L 3$ were significantly associ- 
ated with childhood asthma. It has been suggested that GSDML SNPs modify ORMDL3 expression. Subsequently, Halapi et al. (2010) reported that several sequence variants of 17q21, including rs2305480 and rs8067378, are associated with asthma and affect the expression levels of both GSDML and ORMDL3. ORMDL3 is a member of the gene family that encodes transmembrane proteins anchored in the endoplasmic reticulum, but their function is also unclear (Hjelmqvist et al., 2002). The variants in the ORMDL3 gene associated with asthma have received little attention, and only two SNPs have been investigated: rs4378650 (Galanter et al., 2008; Wu et al., 2009) and rs12603332 (Galanter et al., 2008). Moffatt et al. (2007) initially described $O R M D L 3$ as an asthma susceptibility gene, but none of the associated SNPs in this study are directly located at $O R M D L 3$. An increasing number of researchers have considered that the association between 17q21 and asthma may involve genes other than ORMDL3 in this region (Wjst, 2008; Leung et al., 2009; Halapi et al., 2010). In the present study, high linkage was found between these SNPs in ORMDL3 and GSDML. It is suggested that these variants may be co-regulated in gene expression of GSDML and ORMDL3, contributing to asthma susceptibility. Another SNP, rs8069176 upstream of the GSDML gene, has been found to be associated with asthma in a French population (Bouzigon et al., 2008). This association was not found among all subjects but in male subjects, along with the AA protective effect, similar to the study of Bouzigon et al. (2008).

A study by Galanter et al. (2008) in Mexicans and African Americans showed an association for rs12603332 and rs11650680. However, our data did not show such an association. The reason for this may be the difference in ethnic groups, or it may be that a larger sample needs to be investigated to provide sufficient statistical power to detect the association. This may also explain the discrepancy between our data and those from another study in children from Hong Kong (Leung et al., 2009), in which rs 11650680 was shown to be associated with asthma. Asthma is a complex disease associated with numerous genetic and environmental factors. Our use of adult subjects may have weakened the power to detect significant genetic associations.

In conclusion, we confirmed the existence of genetic associations between asthma and the chromosome 17q21 in a Han Chinese population. Significant associations between two SNPs, rs2305480 and rs8067378 in the GSDML gene, and asthma were found in this study. Although the biological mechanism behind these associations remains unknown, the polymorphisms provide a predictor that may be useful for future targeted research into the prevention of asthma.

\section{ACKNOWLEDGMENTS}

Research supported by Key Programs for the Science and Technology Development of Guangzhou (Grant \#2008A1-E4151) and the Guangdong Natural Science Foundation (Grant \#10151401501000001).

\section{REFERENCES}

Bisgaard H, Bonnelykke K, Sleiman PM, Brasholt M, et al. (2009). Chromosome 17q21 gene variants are associated with asthma and exacerbations but not atopy in early childhood. Am. J. Respir. Crit. Care Med. 179: 179-185.

Bouzigon E, Corda E, Aschard H, Dizier MH, et al. (2008). Effect of 17q21 variants and smoking exposure in early-onset asthma. N. Engl. J. Med. 359: 1985-1994. 
Eder W, Ege MJ and von Mutius E (2006). The asthma epidemic. N. Engl. J. Med. 355: 2226-2235.

Flory JH, Sleiman PM, Christie JD, Annaiah K, et al. (2009). 17q12-21 variants interact with smoke exposure as a risk factor for pediatric asthma but are equally associated with early-onset versus late-onset asthma in North Americans of European ancestry. J. Allergy Clin. Immunol. 124: 605-607.

Galanter J, Choudhry S, Eng C, Nazario S, et al. (2008). ORMDL3 gene is associated with asthma in three ethnically diverse populations. Am. J. Respir. Crit. Care Med. 177: 1194-1200.

Halapi E, Gudbjartsson DF, Jonsdottir GM, Bjornsdottir US, et al. (2010). A sequence variant on 17q21 is associated with age at onset and severity of asthma. Eur. J. Hum. Genet. 18: 902-908.

Hirota T, Harada M, Sakashita M, Doi S, et al. (2008). Genetic polymorphism regulating ORM1-like 3 (Saccharomyces cerevisiae) expression is associated with childhood atopic asthma in a Japanese population. J. Allergy Clin. Immunol. 121: 769-770.

Hjelmqvist L, Tuson M, Marfany G, Herrero E, et al. (2002). ORMDL proteins are a conserved new family of endoplasmic reticulum membrane proteins. Genome Biol. 3: RESEARCH0027.

Leung TF, Sy HY, Ng MC, Chan IH, et al. (2009). Asthma and atopy are associated with chromosome 17q21 markers in Chinese children. Allergy 64: 621-628.

Li X, Yang XX, Hu NY, Sun JZ, et al. (2011). A risk-associated single nucleotide polymorphism of SMAD7 is common to colorectal, gastric, and lung cancers in a Han Chinese population. Mol. Biol. Rep. 38: 5093-5097.

Madore AM, Tremblay K, Hudson TJ and Laprise C (2008). Replication of an association between 17q21 SNPs and asthma in a French-Canadian familial collection. Hum. Genet. 123: 93-95.

Moffatt MF, Kabesch M, Liang L, Dixon AL, et al. (2007). Genetic variants regulating ORMDL3 expression contribute to the risk of childhood asthma. Nature 448: 470-473.

Ober C and Hoffjan S (2006). Asthma genetics 2006: the long and winding road to gene discovery. Genes Immun. 7: 95-100.

Sleiman PM, Annaiah K, Imielinski M, Bradfield JP, et al. (2008). ORMDL3 variants associated with asthma susceptibility in North Americans of European ancestry. J. Allergy Clin. Immunol. 122: 1225-1227.

Tavendale R, MacGregor DF, Mukhopadhyay S and Palmer CN (2008). A polymorphism controlling ORMDL3 expression is associated with asthma that is poorly controlled by current medications. J. Allergy Clin. Immunol. 121: 860-863.

Wjst M (2008). ORMDL3 - guilt by association? Clin. Exp. Allergy 38: 1579-1581.

Wu H, Romieu I, Sienra-Monge JJ, Li H, et al. (2009). Genetic variation in ORM1-like 3 (ORMDL3) and gasdermin-like (GSDML) and childhood asthma. Allergy 64: 629-635. 